\title{
Principes de structuration des pratiques culturelles : stratification et âge
}

Une révision du modèle de La Distinction

Hervé Glevarec et Michel Pinet

\section{OpenEdition}

\section{Journals}

Édition électronique

URL : http://journals.openedition.org/ress/2341

DOI : 10.4000/ress.2341

ISSN : $1663-4446$

\section{Éditeur}

Librairie Droz

Édition imprimée

Date de publication : 1 juin 2013

Pagination : 121-152

ISBN : 978-2-600-01749-7

ISSN : 0048-8046

\section{Référence électronique}

Hervé Glevarec et Michel Pinet, «Principes de structuration des pratiques culturelles : stratification et âge », Revue européenne des sciences sociales [En ligne], 51-1 | 2013, mis en ligne le 01 janvier 2017 consulté le 10 décembre 2020. URL : http://journals.openedition.org/ress/2341 ; DOI : https://doi.org/ $10.4000 /$ ress.2341 


\title{
PRINCIPES DE STRUCTURATION DES PRATIQUES CULTURELLES: STRATIFICATION ET ÂGE
} UNE RÉVISION DU MODÈLE DE LA DISTINCTION HERVÉ GLEVAREC \& MICHEL PINET CNRS - Laboratoire Communication et Politique \& CNRS - Centre Lillois d'Études et de Recherches Sociologiques et Économiques herve.glevarec@lcp.cnrs.fr / michel.pinet@gmail.com

Résumé. Dans le modèle d'analyse des pratiques et des goûts culturels de La Distinction, Pierre Bourdieu soutient que les capitaux économiques et culturels des individus sont d'une part des variables plus déterminantes que d'autres facteurs dits «secondaires» comme l'âge, le sexe ou la résidence et, d'autre part, que leur volume et leur composition permettent de rendre compte du champ des styles de vie. L'analyse des correspondances multiples menée sur les données 2008 de l'Enquête sur les pratiques culturelles des Français du ministère de la Culture confirme que si l'axe de la stratification sociale des pratiques culturelles demeure premier dans la structuration des pratiques culturelles, le second, chez P. Bourdieu celui de la différenciation selon le rapport des possessions culturelles ou économiques, est dorénavant un axe de l'âge. Les travaux longitudinaux qui montrent l'effet générationnel au-delà de l'âge accentuent la thèse qu'un principe de structuration des pratiques culturelles contemporaines réside dans la fréquentation même des biens par une génération historique. Ce facteur prendrait alors la forme d'un tournant culturel dans l'analyse des pratiques culturelles.

Mots-clés: capital culturel, distinction, diversité culturelle, générations, pratiques culturelles.

\begin{abstract}
In the Distinction's model of analysis of practices and cultural tastes Pierre Bourdieu argues that the economic and cultural capitals are, on the one hand, more important variables that secondary factors as age, gender, residence and, on the other hand, that their volume and composition explain the structuration of lifestyles. Since the 1990s, sociologists put forward this second set of variables, including variables of age and generation, as structural ones. The multiple correspondences analysis we conducted on 2008 data from the statistical survey of French cultural practices of the Ministry of culture confirms that, if the axis of social stratification remains first in the structuring of cultural practices, the second is now an axis of age. Longitudinal study highlights the generational effect behind the age, which leads to support a structuring principle of contemporary cultural practices. This dimension would then mean a cultural turn in the analysis of cultural practices.
\end{abstract}

Keywords: cultural capital, distinction, cultural diversity, generation, cultural practices. 


\section{LA SOCIOLOGIE STRUCTURALISTE DES PRATIQUES CULTURELLES}

Pierre Bourdieu a décrit les pratiques culturelles en les rapportant aux «positions sociales » et en y indexant leur valeur sociale (qui est désignée comme leur degré de légitimité). Ainsi deux espaces se superposent-ils: les positions sociales et les biens culturels, dans un rapport d'homologie. Ces espaces ont été décrits comme des plans à deux axes, haut / bas et gauche / droite, structuré pour l'espace des positions par deux sortes de capitaux, économique et culturel, et ordonné pour le second espace par des valeurs de légitimité attachées aux biens. Le modèle théorique en est représenté dans La Distinction sous la forme de «l'espace des positions sociales» et de «l'espace des styles de vie» (Bourdieu, I979, p. I40-I4I).

Le modèle structuraliste de La Distinction se caractérise par deux traits: la dominance des capitaux culturels et économiques sur les autres variables et une détermination double selon le volume et la composition de ces deux capitaux. Le modèle structuraliste à deux axes de la distribution et de la différenciation est articulé par P. Bourdieu à la méthode factorielle d'analyse et de représentation statistique des données de pratiques et de goûts (Le Roux et Rouanet, 20I0). À ce titre, la synthèse de l'information sous formes d'espaces factoriels est l'outil statistique idéal pour toute théorie de l'homologie puisqu’elle permet de considérer l'espace des pratiques et l'espace des facteurs illustratifs comme deux calques qui se superposent (Rouanet, Ackermann et Le Roux, 2000).

Dans La Distinction, Bourdieu recourt pour partie à la méthode de l'analyse factorielle, dans les deux cas des «variantes du goût dominant» et des «variantes du goût petit-bourgeois» (Bourdieu, 1979, p. 296, p. 300, p. 92 et p. 94). En ce qui concerne les dits «espace des positions sociales et espace des styles de vie» ils ne sont pas obtenus par une analyse factorielle. En effet, écrit Bourdieu,

bien qu'il en ait certaines apparences et que l'on se soit aidé de différentes analyses des correspondances pour le construire, bien que nombre d'analyses des correspondances aient produit des espaces qui s'organisent selon la même structure (à commencer par les analyses des données de notre enquête 
qui seront présentées ci-dessous), les schémas présentés ici ne sont pas des diagrammes plan d’analyses des correspondances (ibid., p. I39).

L'espace des positions et des styles ne correspond donc pas aux plans d'une analyse factorielle. Quant aux deux analyses factorielles présentées dans La Distinction, elles sont produites sur une base circonscrite d'individus selon leur catégorie socioprofessionnelle (les «dominants» d'une part, et les «petit-bourgeois» d'autre part). P. Bourdieu indique avoir aussi mené une analyse factorielle sur les classes populaires, mais elle n'apparaît pas dans La Distinction, sans qu'une raison à cette absence soit donnée (ibid. p. 294, note 3 ). ${ }^{2}$ On ne sait donc pas ce qu'une analyse factorielle portant sur la population de l'ensemble des Français aurait produit, soit de proche, soit de différent des dits espaces des positions sociales et des styles de vie.

Cette ambiguïté de l'espace social au cœur de La Distinction, qui malgré les apparences n'est pas le produit graphique d'une analyse factorielle tout en représentant des écarts relatifs entre items et des données chiffrées (par exemple des histogrammes de composition des catégories sociales ${ }^{3}$ ) - ambiguïté scientifiquement préjudiciable (Lahire, I996 ; Mercklé, 20I0) -, permet de rappeler que toute analyse statistique sur les pratiques culturelles procède d’au moins deux choix, l'un qui porte sur le périmètre des variables culturelles et socio-démographiques retenues, l'autre sur le principe actif ou illustratif qui leur est accordé dans l'analyse (laisse-t-on l'espace se structurer par les variables culturelles retenues ou le structure-t-on par des variables socio-démographiques choisies ?) ${ }^{4}$.

I La question demeure de savoir pourquoi cet espace factoriel n'a pas été produit.

2 Une telle remarque n'est pas sans rapport avec la critique du légitimisme du modèle de La Distinction par J.-C. Passeron et C. Grignon qui pointaient l'amoindrissement de la densité descriptive de l'auteur à mesure que son regard portait vers les catégories populaires (Grignon et Passeron, 1989).

3 Quelle valeur statistique a l'affirmation de Bourdieu que sur le diagramme chaque information pertinente «caractérise d'autant plus une catégorie qu'elle figure plus près de son intitulé» (Bourdieu, 1979, p. 143) si ce diagramme n'est pas un espace factoriel?

4 La version parue dans les Actes de la recherche en sciences sociales (Bourdieu et Saint-Martin, 1976) est encore plus problématique puisqu'elle propose de superposer un calque - au sens physique des pratiques à un fond des positions sociales, faisant comme si les deux avaient été produits de façon indépendante par une procédure statistique et informatique, ce qui est encore moins le cas 
Depuis La Distinction, les analyses factorielles sur les pratiques culturelles ont été répétées, sur la base de l'ensemble des Français, ou encore sur la population des jeunes, avec les pratiques en variables actives, ensuite éclairées par les variables socio-démographiques (Donnat et Cogneau, I990; Donnat, I994; 1998 ; Donnat et Tolila, 2003 ; Donnat, 2004 ; Octobre, 2004 ; Coulangeon et Lemel, 2007 ; Donnat, 2009; Octobre, Detrez, Mercklé et Berthomier, 20 I I). En regard de la structuration mise en avant par P. Bourdieu dans La Distinction, les travaux ultérieurs, centrés sur les pratiques culturelles et non sur l'ensemble des styles de vie, soulignent deux différences : d'une part, la mise en évidence de l'exclusion culturelle et, d'autre part, l'amendement de l'homologie entre «position sociale» (entendue comme coordonnées sur la carte des capitaux) et univers de pratiques culturelles.

Les analyses postérieures à La Distinction ont pour caractéristiques de faire apparaître un premier axe de structuration, en quelque sorte absent du modèle bourdieusien, même s'il est tout à fait intégrable dans celui-ci, du fait qu'il porte sur les styles de vie en général, celui de la participation culturelle, en termes de «réclusion» ou «d'exclusion» (Donnat et Cogneau, 1990).5 La sociologie des « univers culturels » menée au sein du ministère de la Culture met en première place un critère de différenciation par la fréquentation même, celui qui sépare les pratiquants culturels des «exclus» (Donnat, 1994). Un premier principe identique de structuration se constate dans l'analyse des pratiques et des goûts culturels des Anglais menée par Tony Bennett et al. (2009). Le premier axe de

qu'avec la version à plat de La Distinction. Le calque des Actes de la recherche en sciences sociales, outil de la thèse de l'homologie, est en effet encore plus faux que le schéma à plat puisqu'il sépare deux espaces de données à partir de ce qui est indiqué être une schématisation non issue d'une procédure statistique. II n'y a pas besoin d'être grand épistémologue pour voir que Bourdieu franchit là un interdit scientifique, celui de construire un lien de dépendance entre des séries de données non issues d'une procédure statistique (et a fortiori la même).

5 L'annexe I de La Distinction consacrée à la méthode indique que la restriction du champ de l'enquête aux pratiquants est à la fois délibérée (les exclus de la culture sont alors les agriculteurs), justifiée et cohérente avec le modèle. «On a, d'autre part, écrit P. Bourdieu, exclu de l'analyse les agriculteurs et salariés agricoles au terme d'une pré-enquête qui avait fait conclure à l'inadéquation totale du questionnaire et à la nécessité de recourir à de tout autres méthodes pour saisir les dispositions d'une population totalement étrangère à la culture légitime et même, pour l'essentiel, à la culture moyenne» (Bourdieu, 1979, p. 589). 
leur analyse des correspondances multiples recouvre la dimension du degré de l'engagement dans la culture. Les axes suivants mettent en avant successivement la distinction entre pratiques culturelles contemporaines ou commerciales et pratiques classiques, pratiques d'intérieur et pratiques d'extérieur et, enfin, entre les pratiques selon leur degré d'intensité (voracious vs moderate) (Bennett, Savage, Silva, Warde, Gayo-Cal et Wright, 2009, p. 44).

Relativement au modèle dit de «l'homologie structurale» entre «positions sociales» et univers de pratiques et de goûts culturels, Olivier Donnat avançait dès les années 1990 trois éléments au nombre des raisons qui «interdisent aujourd'hui de référer mécaniquement les univers culturels aux milieux sociaux»: en premier lieu, «les catégories socioprofessionnelles sont aujourd'hui moins clairement identifiables à travers les seuls niveaux de ressources et de qualification professionnelle, et surtout moins homogénéisées par des appartenances visibles. » En second lieu, «les facteurs de différenciation internes sont devenus plus puissants. [...] À bien des égards, la société française apparaît aujourd'hui comme la somme de trois générations - celle de l'avant-guerre, celle des baby-boomers et celle de la démocratisation scolaire et de la banalisation de l'audiovisuel. »Et, enfin, «le bouleversement des conditions d’accès au savoir et aux œuvres et la montée en puissance de l'économie médiatico-publicitaire ont favorisé une hybridation croissante des univers culturels, notamment dans les générations nées après-guerre » (Donnat, 1994, p. 345). ${ }^{6}$ En effet, dès la fin des années 1980, l’analyse sociologique met en avant la variable de l'âge: «l'espace social des loisirs a des lignes de force sociales, écrit Denis Cogneau: le capital culturel en premier lieu, puis l'âge, puis le sexe» (Cogneau, I990, p. 202). Mais cette variable n’a pas la dimension «générationnelle » qu'elle a acquise dorénavant :

6 Dans le domaine musical, P. Coulangeon et Y. Lemel remettent aussi en question «l'homologie sociale biunivoque» des goûts: «Ces caractéristiques [celles indiquées par l'analyse factorielle des données de l'enquête EPCV 2003] éliminent de façon nette toute tentative de cartographier la distribution du goût musical en termes d'une homologie sociale biunivoque: la musique savante n'est pas plus une musique des catégories supérieures que la musique pop la musique des catégories inférieures» (Coulangeon et Lemel, 2007, p. 98-99). 
Après 25 ans, la position dans l'espace des loisirs dépend directement de la position dans la hiérarchie sociale et de la fraction de position sociale. [...] Avant 25 ans [...], l'arbre [la classification hiérarchique] met en revanche en lumière une homogénéité culturelle entre "adolescents” de toutes origines (ibid., p. 276).

L'analyse demeure prudente sur ce point puisque la variable de l'âge y est un effet de cycle de vie (l'adolescence), bien qu'effet historiquement situé dans la décennie i960?

En 20ı I, O. Donnat revient sur ce point à l'appui d'une comparaison des données antérieures qui permet de faire apparaître la variable de la génération, comparaison précédée elle-même par la publication de L’Approche générationnelle des pratiques culturelles et médiatiques en 2007 (BIPE/DEPS, 2007). Les «mutations générationnelles » apparaissent comme la seconde force de structuration des pratiques face à «la permanence d'une forte stratification sociale des pratiques culturelles » qui confirme, aux yeux d'Olivier Donnat, la pertinence des schémas théoriques articulés autour de la notion de capital culturel. O. Donnat en conclut que «l'évolution des pratiques culturelles [entre i973 et 2008] doit être appréciée d’un double point de vue difficilement conciliable» (20I I, p. 28). Si, à la faveur de la place structurante prise par la génération, variable secondaire, les transformations historiques affectent un modèle initialement positionnel, elles n'ont pas entraîné la révision conceptuelle qu’elles semblent pourtant impliquer. En effet, ce qui apparaît «difficilement conciliable» l'est au regard des catégories intellectuelles que les sociologues mobilisent, le réel des pratiques les conciliant, lui, parfaitement; la difficulté sociologique et théorique réside en effet dans l'accord entre une détermination de la stratification par le «capital culturel» et une détermination par une dimension qui explique la variation générationnelle. Dans Les Français face à la culture, O. Donnat nommait cette seconde variable «capital informationnel», à savoir les connaissances possédées par un individu, mesurées au nombre d’artistes connus, connais-

7 «Nous nous abstiendrons de toute prévision macrosociale sur le poids que peut faire peser sur l'espace culturel et dans les années à venir cette culture adolescente. Elle nous a paru toutefois ne pas devoir remettre en question ni la position élitaire de la culture cultivée ni la division sexuelle du loisir» (Cogneau, 1990, p. 286). 
sances différenciées en «modernes» et «classiques» selon le rapport entre nombre «d'artistes consacrés connus» et nombre «d'artistes consacrables connus » (Donnat, 1994, p. 72-73). ${ }^{8}$ Ainsi, avoir une structure moderne du capital informationnel suppose, en 1988, de connaître le chanteur Renaud, le musicien Miles Davis ou le cinéaste Jean-Luc Godard et une structure classique de connaître le compositeur Pierre Boulez, le sculpteur Auguste Rodin ou le metteur en scène Jean Vilar. De façon décisive O. Donnat (ibid., p. 80) distingue là un capital informationnel d'un capital culturel (qu'il identifie, comme le font couramment les sociologues, au diplôme). À la différence du capital culturel, qui est acquis en famille ou à l'école, un tel capital informationnel «d'artistes consacrables » œuvrant dans des genres en voie de reconnaissance s'acquière en effet hors de ces deux espaces traditionnels de socialisation. ${ }^{9}$

Les «mutations générationnelles» sur lesquelles insiste O. Donnat signalent qu'un facteur de fréquentation culturelle passée et contemporaine structure dorénavant les pratiques culturelles des Français.

Le deuxième enseignement majeur concerne l'importance de la dimension générationnelle dans la plupart des mutations observées, qu'il s'agisse de l'augmentation de la durée d'écoute de la télévision, du boom musical, de la baisse de la lecture d'imprimés, des progrès des pratiques artistiques en amateur ou des transformations des comportements en matière de spectacle vivant. Chaque fois qu'il y a eu changement, celui-ci a été initié par la génération montante avant d'être amplifié par les suivantes, qui ont conservé en vieillissant une grande partie des habitudes acquises au temps de leur jeunesse (Donnat, 20I I, p. 28).

8 En 1990, D. Cogneau parle «d'atout culturel des âges jeunes》: «l'espace des loisirs et la diversité des rapports à l'extérieur de chez soi peuvent se découper selon trois lignes de forces sociales: le capital culturel, l'atout culturel des âges jeunes et les différences sexuelles» (Cogneau, 1990, p. 20I). Un «atout culturel» est-il un «capital»? Là est la question sociologique.

9 Dans ce livre 0 . Donnat note «la cohérence globale qui existe entre le volume de capital informationnel des individus et leur degré de participation aux activités culturelles» (1994, p. 325). Nous avancerons l'idée qu'il y a une forme de marche arrière, ou une hésitation, quant au modèle adopté, entre 1994 et 2012, qui du «capital informationnel» revient au «capital culturel» alors même que la structuration par la génération culturelle s'accentue. 
La fréquentation culturelle passée et contemporaine indique qu'un facteur culturel - avoir rencontré par le passé ou fréquenté actuellement tel bien culturel - participe dorénavant de l'explication des pratiques actuelles. Il s'agit là d'une rectification de l'affirmation que «c'est le volume et la structure du capital [qui] donnent leur forme et leur valeur spécifiques aux déterminations que les autres facteurs (âge, sexe, résidence, etc.) imposent aux pratiques » (Bourdieu, 1979, p. I19). Dans le modèle de La Distinction, les capitaux économiques et culturels mobilisés par Bourdieu sont en effet des variables distinctes de l'âge, du sexe, de la résidence, etc. Ce sont les variables de ce second ensemble qui sont mises en avant par les sociologues à partir des données d’enquête récentes. Il convient d’ajouter qu’O. Donnat date la valeur prise par cette «variable générationnelle» du début des années 1970.10

$\mathrm{Au}$ regard de ces tensions entre évolution de la structuration des pratiques culturelles et modèle d'analyse structuraliste en sociologie de la culture, nous nous proposons, sur la base des données de l'enquête pratiques culturelles 2008 du ministère de la Culture, de donner à voir la distribution contemporaine des pratiques des biens culturels et d'évaluer à nouveaux frais la valeur des facteurs en jeu, en premier lieu celle du «capital culturel». Une telle réflexion permet de soutenir un changement de l'interprétation structuraliste, positionnelle, du rapport à la culture.

\section{APPRÉHENDER LE CHAMP DES PRATIQUES CULTURELLES CONTEMPORAINES}

\section{I. LA MÉTHODOLOGIE STATISTIQUE DE L'ANALYSE DES CORRESPONDANCES MULTIPLES ET DE LA CLASSIFICATION HIÉRARCHIQUE}

L'enquête sur les pratiques culturelles des Français contient davantage de variables de pratiques (de fréquence ou de volume de pratiques) que de variables de goût (d’appétence ou d’inappétence), à la différence de l'enquête récente menée par Tony Bennett et ses collègues en Angleterre. L'enquête du ministère de la Culture est en effet marquée d’un héritage et d’un para-

10 «Les résultats de l'analyse rétrospective que nous avons présentés [...] soulignent l'ampleur du renouvellement des pratiques culturelles depuis le début des années 1970»(Donnat, 201 I, p. 28). 
digme que l'on peut désigner sous l'expression d'accès différentiel à la culture (Glevarec, 20I3; Glevarec et Pinet, 20I3). Le terme même de pratique signale une insistance sur la participation et sa fréquence, que la valeur donnée aux pratiques amateur n’a que peu infléchi, plutôt que sur ce qui pourrait être désigné comme des activités culturelles (Donnat, 2005).

Sur le plan méthodologique, le défi de l'analyse statistique des pratiques et des goûts culturels menés à partir d'une enquête existante est de rendre compte de la diversité des «bouquets de pratiques», entendus comme la combinaison pour chaque enquêté de ses rapports, existants ou non, avec le panel des pratiques retenues dans l'enquête (qui couvrent un large champ: la musique, la lecture d'ouvrages ou de presse, les différentes formes du spectacle vivant, le cinéma, le rapport aux musées et au patrimoine, les pratiques amateur, l'écoute des médias, radio et télévision, mais aussi les pratiques liées aux nouveaux supports, internet, ordinateurs et consoles). Nombreuses sont les combinaisons possibles de pratiques et plurielles les façons de synthétiser l'information statistique et d'en proposer une figuration.

L'analyse des correspondances multiples sur les pratiques culturelles couplée à la classification hiérarchique permet, d'une part, de faire apparaître les lignes d'opposition qui structurent un champ d'activités et offre, d'autre part, la possibilité de la comparaison avec l'analyse pionnière de Bourdieu. La classification hiérarchique apparaît comme la méthode quantitative la plus adaptée à l'approche en termes de différenciations. En effet, dans l'univers en apparence continu des ensembles de pratiques, elle permet de faire émerger, au travers des classes et de l'arbre de leur regroupement, des îlots de plus forte densité où les individus partagent cet ensemble de pratiques (ou, à tout le moins, d'écarts à la moyenne dans les pratiques). Sa logique revient à travailler sur une «distance» entre les vecteurs de pratiques individuels (ensemble des pratiques de chaque individu), dont on puisse faire l'instrument d'une partition de l'ensemble de la population en «groupes» de pratiquants culturels, de telle sorte qu'on se ressemble (sous l'angle du bouquet de pratiques) le plus possible au sein d'un même groupe et le moins possible d'un groupe à l'autre. L'objectif d'une telle analyse est de regrouper les individus de l'échantillon en classes les plus 
homogènes possible - du point de vue du critère de ressemblance défini plus haut -, les classes étant entre elles le plus dissemblables possible. La procédure «regroupe» d'abord les individus les plus ressemblants, puis progressivement, par itération, les individus (ou groupes déjà constitués) les plus proches, pour arriver au final à une «classe unique » comportant tous les individus.

Cette procédure de regroupement progressif peut être représentée par un arbre qui rend compte de la succession des regroupements dans l'algorithme de classification sous la forme d'un dendrogramme qui figure un peu plus loin (Figure 3). Une classification en n classes, du point de vue de cet arbre, correspond à une coupe à l'endroit où l'arbre comporte n branches. Cette représentation de type généalogique illustre la manière dont on passe d'une partition encore proche de l'état non regroupé initial (le bas du graphique) au regroupement ultime en une seule classe (haut du graphique). De cette manière, il est possible de voir dans les emboîtements amont et aval à la fois ce qu'une classe réunit et ce qui peut l'éloigner ou la rapprocher de telle ou telle autre classe. Elle représente l'outil pertinent pour identifier ces archipels de goûts qui constituent un des piliers de notre approche en termes de combinaisons circonscrites des préférences et des pratiques, modélisation que nous avons désignée sous le terme de «tablature des goûts » (Glevarec et Pinet, 2009 et 20I2).

\subsection{LES DOMAINES CULTURELS DE L'ANALYSE SUR LES PRATIQUES CULTURELLES}

Nous avons mené une analyse des correspondances multiples sur les données de l'enquête Pratiques culturelles des Français de 2008 réalisée par le ministère de la Culture. ${ }^{.1}$ Le champ culturel des pratiques que nous avons retenues est celui disponible dans l'enquête et son périmètre similaire à celui habituellement considéré.

II Le champ des variables culturelles retenues est indiqué en annexe, Tableau 3. Toutes les annexes au présent article sont accessibles en ligne depuis la page de l'article sur le site de Revues.org et téléchargeables à cette adresse: http://ress.revues.org/2346. 
La base contient 4824 individus, I 88 variables actives possédant 493 modalités associées et 43 variables illustratives possédant I89 modalités associées. Le seuil de I \% d'effectifs en-dessous duquel la procédure redistribue une modalité trop rare dans les autres modalités est relativement faible au regard des usages, mais permet de situer dans les espaces factoriels, certes avec prudence, certaines modalités très minoritaires mais à forte signification sociologique. En ce qui concerne le tri des individus, nous avions d’abord retenu la totalité des 5004 individus de l'enquête, mais les premières analyses exploratoires ont montré un rôle très polarisant, pas seulement sur un axe, d'un petit groupe d'individus (I80 personnes) cumulant des «non réponses» sur un nombre important de questions. Nous avons préféré écarter de l’analyse ces I80 personnes et les mettre en «individus supplémentaires » pour ne pas perturber inutilement l'interprétation des espaces par un élément relevant plutôt de l'artefact d'enquête. L'analyse porte donc au final sur 4824 individus actifs.

Les I89 variables actives couvrent une diversité de domaines culturels : jeux vidéo (7 variables), ordinateur et internet (2 variables), télévision (4I variables), radio (I9 variables), musique (I3 variables), lecture des informations (presse et écrans) (i6 variables), lecture d'œuvres et de documents (d’ouvrages, écrans) (19 variables), spectacle vivant (I4 variables), cinéma (I8 variables), musées (8 variables), expositions, parcs et patrimoine (I I variables) et activités en amateur (20 variables). ${ }^{2}$ Les 43 variables illustratives retenues portent sur un ensemble de pratiques relatives aux préférences en termes de loisirs, de temps libre, de sorties le soir, au téléchargement sur internet (I8 variables) et sur certaines pratiques pour lesquelles les effectifs étaient trop faibles pour être retenus dans les variables actives et, enfin, aux variables socio-démographiques (I5 variables) et à l'équipement médiatique du foyer (Io variables).

12 Quelques variables de pratiques, redondantes avec d'autres ou aux effectifs trop faibles, ont été placées en variables illustratives. Certaines modalités n'ont pas contribué à l'analyse puisque les petits effectifs qu'elles concernaient (inférieures à 50 personnes) ont été ventilés de façon aléatoire sur les autres modalités. 
Tableau I. Contribution de chaque domaine culturel aux différents axes de l'analyse en correspondances multiples

\begin{tabular}{|l|c|c|c|c|c|}
\hline \multicolumn{1}{|c|}{ DOMAINES CULTURELS } & AXE I & AXE 2 & AXE 3 & AXE 4 & AXE 5 \\
\hline ACTIVITÉS AMATEUR & 12.9 & 2.8 & 7.9 & 7.1 & 15.9 \\
\hline CINÉMA & 6.8 & 8.5 & 4.5 & 5.1 & 10.7 \\
\hline JEUX VIDÉO & 6.1 & 9.1 & 1.0 & 2.4 & 0.7 \\
\hline LECTURE DES INFORMATIONS (presse et écrans) & 15.2 & 4.6 & 3.3 & 23.6 & 5.1 \\
\hline LECTURES D'OUVRAGES (y.C. sur écrans) & 12.0 & 10.1 & 7.0 & 3.4 & 8.4 \\
\hline MUSÉE & 4.8 & 7.3 & 0.4 & 3.3 & 1.4 \\
\hline MUSIQUE & 9.0 & 14.1 & 8.4 & 7.4 & 3.3 \\
\hline ORDINATEUR ET INTERNET & 5.7 & 0.8 & 1.3 & 8.8 & 2.8 \\
\hline EXPOSITIONS, PARCS ET PATRIMOINE & 8.4 & 13.1 & 7.1 & 6.8 & 6.7 \\
\hline RADIO & 3.0 & 6.5 & 9.6 & 4.7 & 8.0 \\
\hline SPECTACLE VIVANT & 8.1 & 9.0 & 6.2 & 3.7 & 6.8 \\
\hline TÉLÉVISION & 8.0 & 14.4 & 43.5 & 23.7 & 30.2 \\
\hline
\end{tabular}

Source:enquêtePratiquesculturelles des Français 2008,DEPS,ministèredelaCulture/Clersé/LCP-CNRS. Champ: I 89 variables actives; $N=4824$

L’analyse de correspondances permet d’apprécier la contribution de chaque domaine culturel aux cinq axes (Tableau I ${ }^{13}$. Les axes sont relativement différenciés selon le(s) domaine(s) culturel(s) de pratiques et de goûts. ${ }^{14}$ Les activités en amateur, la lecture des informations et des ouvrages ou documents sont associés pour définir l’axe I, un axe de participation médiatique fortement associé à internet. La musique, les expositions, parcs et patrimoine, la lecture d'ou-

13 Rappelons qu'un axe de rang élevé dans une telle analyse est une dimension (du nuage des points représentant les réponses) sur laquelle le nuage s'étale en révélant une polarisation particulièrement importante. Le premier axe révèle ainsi quelle est la plus forte opposition qui structure un tel ensemble de points, à ses deux extrémités, et ainsi de suite. Tracer le graphique des plans I-2 revient à prendre la photographie du nuage multidimensionnel sous un angle qui donne la meilleure idée possible (à deux dimensions) de la forme globale de ce nuage, révélant ce qui s'y oppose principalement.

14 Les cinq premiers axes expliquent 13,7\% de la variance globale du modèle (le premier axe en explique 6,2\% à lui seul). 
vrages, les jeux vidéo et le spectacle vivant contribuent à l'axe 2. Sur l'axe 3, la télévision domine très largement. L’axe 4 associe la lecture des informations, les pratiques informatiques et internet et la télévision. Enfin, ce sont la télévision, les activités en amateur et le cinéma qui contribuent le plus à définir l’axe 5 .

\section{STRATIFICATION SOCIALE ET DIFFÉRENCIATION SELON L'ÂGE DES PRATIQUES}

\section{I. DIFFÉRENCIATIONS PARTICIPATIVES ET CULTURELLES}

Le premier axe factoriel est un axe de la participation culturelle numérique et contemporaine (voir Figure 4 en annexe, en ligne: http://ress.revues.org/2346). ${ }^{15} \mathrm{Ce}$ premier axe a pour principe d'opposer des pratiques culturelles effectuées sur l'ordinateur ou internet, mais aussi des pratiques de visite de musée, d'écoute de la musique rock, de fréquentation du cinéma et de lecture de bandes dessinées, à un défaut de pratiques. L'opposition majeure se fait entre des «inclus » et des «exclus». Si ce qui a trait aux pratiques sur l'ordinateur et sur internet paraît, à l'examen des items les plus significatifs, dominer l'explication, un examen plus attentif montre que la pratique multiforme de toutes les variantes de l'activité «numérique» se fait en redondance avec les formes classiques de culture, tandis que la distance à l'internet ou aux ordinateurs est associée à une absence de pratiques culturelles de toutes sortes.

Symboliques de ce rapport familier au monde numérique d'un côté de l’axe I sont les deux valeurs-test les plus fortes de ce côté de l'axe, l'usage d'internet pour «l'activité documentaire» (dont la valeur-test est égale à 48,4, soit la plus forte de toute l'analyse tous axes confondus) et la fréquentation de blogs (valeur-test $=44,5$ ). Internet, d'usage quotidien, est omniprésent (usage de Youtube, presse, musique, politique, voyages, vie quotidienne), mais c'est aussi l'ordinateur qui sert à diverses activités (musique, films, écriture, dictionnaires, pratiques graphiques et créatives, etc.). Du point de vue sociodémographique, ce premier axe oppose des catégories de jeunes adultes diplômés à des catégories populaires et âgées.

I5 La mention complète des étiquettes des points est indiquée dans le tableau 3 en annexe. Les signes accompagnant les modalités de goûts expriment l'appétence pour (+) ou l'inappétence pour (-). 
Le second axe est un axe des biens culturels (Figure 5 en annexe, en ligne: http://ress.revues.org/2346). Il oppose une pratique de la culture classique entendue comme la fréquentation des musées, des livres d’art, des essais et de la musique classique d'une part, à une fréquentation de la culture contemporaine des jeux vidéo, de la musique rap et électronique et de la télévision de divertissement d'autre part. Ce second axe oppose des catégories supérieures, diplômées, de 50 ans et plus aux catégories d'étudiants, de jeunes et de célibataires.

Le troisième axe (Figure 6 en annexe, en ligne : http://ress.revues.org/2346) est un axe des médias audiovisuels traditionnels qui oppose une pratique médiatique de la télévision et de la radio, mais aussi des goûts musicaux portés vers la chanson française et internationale et des sorties, notamment familiales, à une absence de fréquentation des médias audiovisuels. Du point de vue sociologique, ce troisième axe oppose un modèle familial d'organisation du temps culturel fortement tourné vers une vie sociale active mais concentrée sur les proches (invitations dans le cercle de la famille et des amis) et l'espace domestique, orienté par une forte présence de la télévision (foyer multi-équipé, goût pour les émissions de talk-show et de télé-réalité), à un pôle de personnes isolées sortant très peu. Il s'agit d'une opposition entre un pôle féminin et populaire, avec présence de jeunes enfants, et un pôle à dominante masculine, où se rejoignent dans une même distance à toute activité extérieure deux profils de personnes isolées à faibles ressources, jeunes étudiants derrière leur écran et veufs à faible vie sociale et culturelle.

Le quatrième axe est un axe des sorties vs des pratiques domestiques de nature numérique. Il oppose une culture de la sociabilité en extérieur à une culture de la domesticité liée à la pratique de l'internet. Il oppose deux catégories particulières qui se distinguent surtout par le rapport aux pratiques numériques (et sans doute, en amont, par le temps disponible...) : d’un côté, dans les valeurs positives de l'axe, des femmes jeunes ou d'âge moyen, mères de famille ayant de jeunes enfants ou des adolescents, peu concernées par les pratiques numériques et internet ou par la presse, mais ayant quelques formes de loisirs extérieurs; de l'autre des retraités, plutôt d'origine populaire ou moyenne, qui 
sortent peu, cumulent les formes d'usage d'internet et privilégient les activités d’information, numériques ou non.

Les trois premiers axes de l'analyse des pratiques culturelles des Français ne sont pas sans une parenté forte avec la description que T. Bennett et al. font des pratiques et des goûts culturels des Anglais en 2003-2004 : l'axe I de leur analyse des correspondances multiples principale n'oppose ainsi pas culture classique et culture populaire, mais participation culturelle et non-participation, et il est structuré par le niveau d'éducation, la position professionnelle et le revenu; l'axe 2, qui oppose culture classique et culture contemporaine est structuré par l'âge; et, enfin, l'axe 3 distingue des pratiques domestiques et des pratiques de sorties et est structuré par le genre (Bennett, et al., 2009, p. 56). Une structuration similaire organise les pratiques culturelles des Belges de Flandre selon le volume du capital pour l'axe I et l'âge pour l'axe 2 (Roose, Van Eijck et Lievens, 20I2).

\subsection{LA DISTRIBUTION PAR L'ÂGE DES PRATIQUES}

L'espace factoriel des variables socio-démographiques (traitées en illustratif dans l'analyse) que donne à voir la figure i possède des points communs avec l'espace factoriel, circonscrit aux catégories supérieures, des «variantes du goût dominant» de La Distinction (Bourdieu, I979, p. 296); la différence - à souligner - réside dans l'orientation prise ici par le niveau de rémunération qui suit celui du diplôme tandis qu'il lui est orthogonal dans l'analyse des données de 1963 (complétées de secondes recueillies en 1967-1968) par Bourdieu (ibid., p. 296 et p. 587-588). En revanche, ce qui apparaît dorénavant quasi-orthogonal à l'axe de la stratification recouvre, dans l'espace contemporain, essentiellement l'âge. ${ }^{16}$

16 P. Bourdieu opère dans La Distinction sur la base très vraisemblable des Français de 18 ans et plus (rien n'est indiqué dans la partie méthodologique à ce sujet) et sur la variable de «chef de ménage ( (comme en témoigne en creux l'absence d'une catégorie comme celle de femme au foyer dans les caractéristiques de l'échantillon; Bourdieu, 1979, p. 590). Nous opérons, dans le cas présent, sur l'individu avec de surcroît une catégorie «interclassiste» «lycéens-étudiants». 
Il convient de noter que dès 1989-1990 (date de recueil de la troisième enquête sur les loisirs des Français), l'analyse de l'espace des loisirs par O. Donnat et D. Cogneau (1990) laissait déjà apparaître, à côté d'une première opposition entre rapport cultivé au monde extérieur et «réclusion», corrélée à la catégorie socioprofessionnelle et aux diplômes, une deuxième opposition entre les très jeunes (et leurs pratiques des boîtes de nuit, du rock vu à la télévision) et les individus plus âgés et diplômés (qui avaient des pratiques classiques de type «sortie à l'opéra », regardaient des émissions culturelles) ainsi qu'une troisième opposition entre univers féminin et univers masculin.

Les transformations par rapport à l'espace social dessiné par P. Bourdieu sont ici de deux ordres: un premier axe des pratiques culturelles demeure structuré par le volume du capital possédé (sa composition culturelle vs économique s'efface, les deux ressources variant ici dans le même sens) ${ }^{17}$; un second axe apparaît structuré par l'âge des individus et renvoie donc au rôle pris par ce facteur en tant qu'il distingue les individus par leur contact différentiel avec la culture. La nouveauté vient de ce que la distribution des biens culturels par l'âge est devenue un facteur structurant, non plus extérieur ou indépendant, du champ des pratiques. L'enquête de 2008 ne permet pas de faire le départ entre les deux variables de l'âge et de la génération puisqu'elle n'est ni longitudinale, ni ne contient de questions rétrospectives sur les pratiques et les goûts. On peut s'appuyer ici sur les analyses longitudinales qui mettent à jour la dimension générationnelle que recouvre la variable de l'âge (BIPE/DEPS, 2007 ; Donnat, 20II).

17 Cette absence du principe de division, culturelle vs économique, de l'espace social, et la congruence des deux variables précitées, est expressément pointée à propos des pratiques et goûts musicaux par C. Coulangeon et Y. Lemel (2010, p. 107). 
Figure I. L'espace des variables socio-démographiques illustratives : Plan factoriel des axes i et 2, modalités à cos2 élevé (seuil de 10\%)

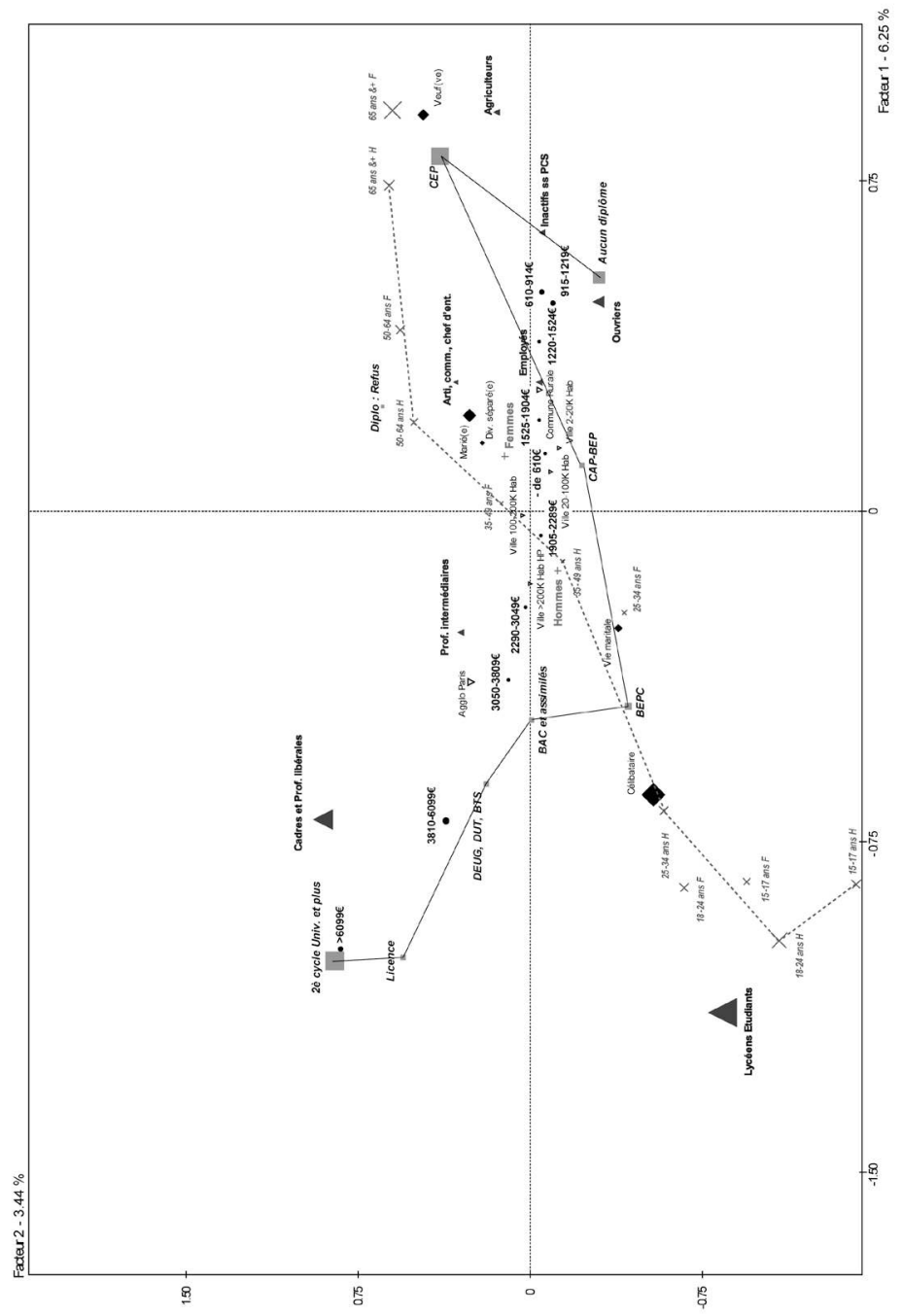

Les symboles sont proportionnels aux cosinus carré des modalités. En euros sont indiqués les revenus bruts mensuels du foyer. 
L'axe 2 de l'analyse des correspondances tel qu'il apparaît caractérisé par les variables socio-démographiques dans la figure I fait figure de nouveauté dans la distribution des pratiques culturelles. Il suggère, dans le prolongement des travaux récents qui mettent en valeur la place prise par le facteur de l'âge puis, grâce aux comparaisons de données sur 30 ans, par la «dynamique générationnelle», de représenter de façon plus détaillée, la composition des variables de professions, de niveau de diplôme et d'âge. La figure 2 a pour but de faire apparaître de façon plus explicite cette structuration des pratiques culturelles contemporaines selon les deux axes de la stratification et de la génération. Elle consiste à projeter sur les deux premiers axes de l'analyse une «position sociale » correspondant au croisement de la profession, du niveau de diplôme et de la génération des individus, ceci pour les catégories d'ouvriers-employés, de professions intermédiaires et de cadres et professions libérales. La représentation qui est donnée à voir rejoint ce que disait $\mathrm{O}$. Donnat dans Les Français face à la culture au mitan des années 1990: «désormais, la proximité générationnelle peut être plus forte que l'origine ou l'appartenance sociale » (1994, p. 345) comme facteur explicatif des pratiques culturelles; il ajoutait: «la société française apparaît aujourd'hui comme la somme de trois générations - celle de l'avant-guerre, celle des babyboomers et celle de la démocratisation scolaire et de la banalisation de l'audiovisuel » (ibid.). L'espace des pratiques culturelles se trouvent ainsi structuré par deux axes principaux de déterminations sociologiques, qui sont un axe du volume du capital (économique et culturel) et un axe de la valeur de l'âge. 
Figure 2. L'espace des positions sociales. Projection sur le plan factoriel des axes I et 2 d'un croisement Profession/Diplôme/Génération*

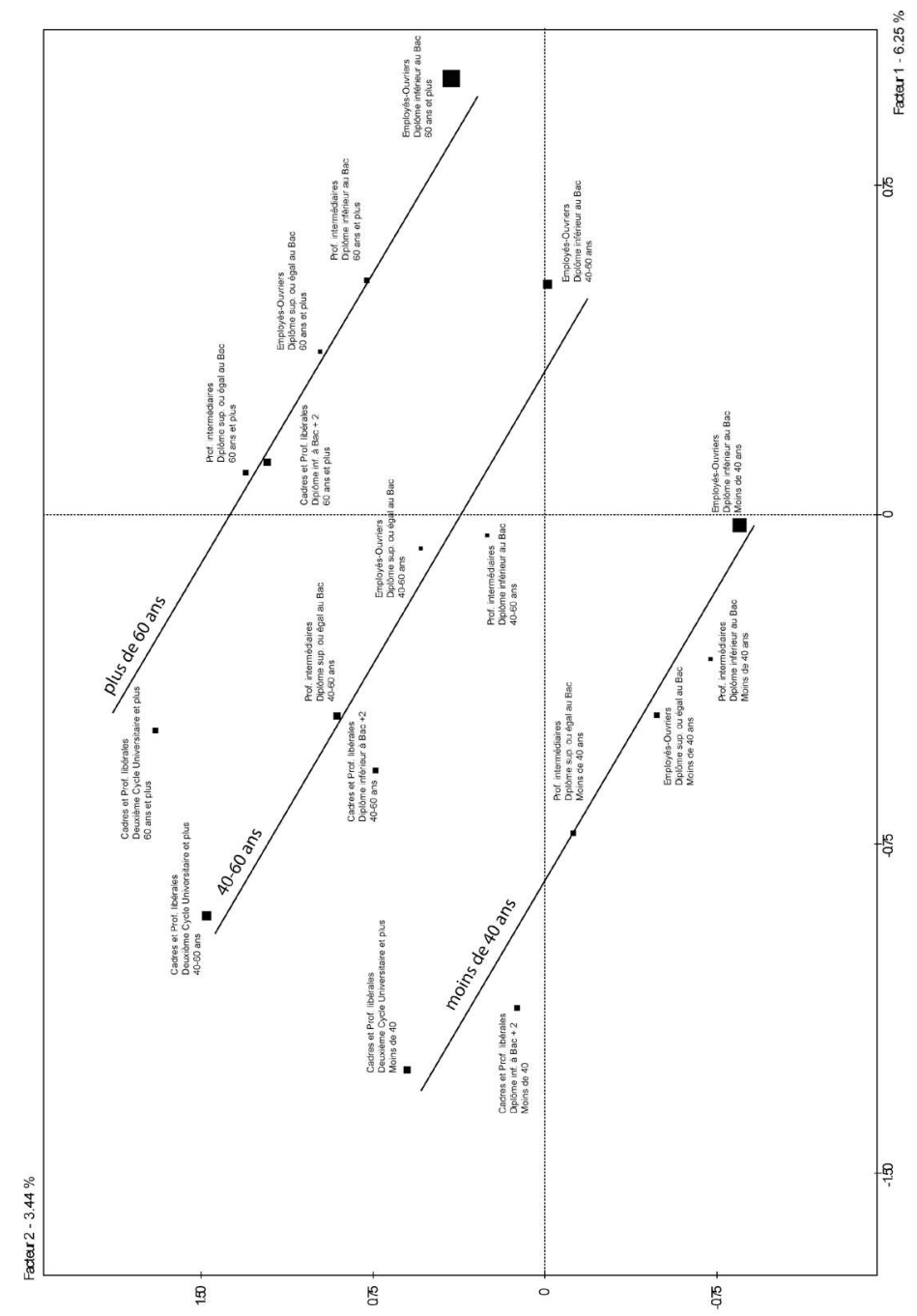

Les symboles sont proportionnels aux cosinus carré des modalités.

* Cet espace représente, par choix, certaines catégories; n'y figurent pas les agriculteurs, les artisans, commerçants et chefs d'entreprise, les lycéens et étudiants et les inactifs. Les retraités sont ici rattachés à leur dernière PCS d'actifs. 


\section{LES UNIVERS DE PRATIQUES SELON LES GENRES CULTURELS}

Les deux axes de la position socioprofessionnelle et du diplôme d'une part et de l'âge d'autre part sont les principaux déterminants du paysage des pratiques culturelles. Afin de qualifier les univers de pratiques dans cet espace contemporain, nous avons procédé à une classification hiérarchique de données qui permettent de différencier des ensembles de pratiques et les caractéristiques de leurs participants.

L'arborescence de la classification hiérarchique qui s’appuie sur les dix premiers axes de l'analyse factorielle laisse voir une coupure forte entre les trois classes les plus à gauche et les trois autres (les deux dernières branches ne fusionnent qu'à l'ultime étape du processus). Nous avons retenu une coupure de l'arbre en six classes. Les modalités les plus distinctives des trois premières classes correspondent presque toujours à un surcroît de certaines pratiques (plutôt qu'à des absences), et surtout les pratiques culturelles liées aux nouvelles technologies et particulièrement à l'internet jouent un rôle important et même parfois hégémonique. Exactement à l'inverse, les trois classes de la droite du graphique se définissent plus souvent par les pratiques qui sont absentes, et par le faible rôle des pratiques de nature numérique.

Figure 3. Dendrogramme de la classification hiérarchique

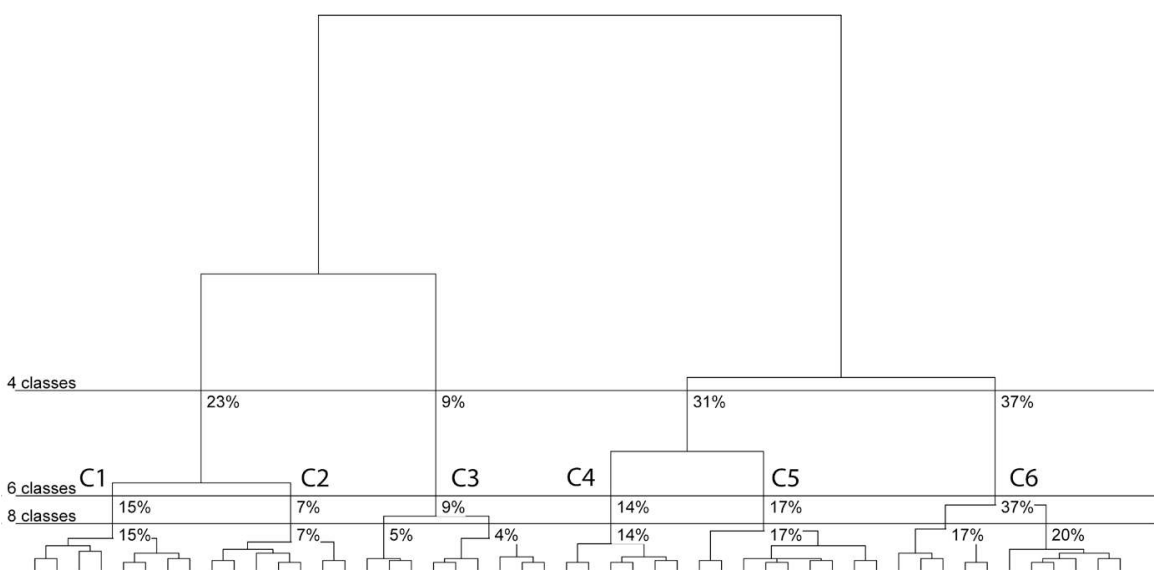

Classification hiérarchique directe; les pourcentages indiqués correspondent à la taille des classes à chaque niveau de coupure. 
La première des trois classes de la gauche de l'arbre de la classification $\left(\mathrm{C}_{\mathrm{I}}\right)$ est sans ambiguïé la classe d'un modèle jeune et même très jeune (fortes valeur-test pour les classes d'âge I5-17 ans et I8-24 ans) de pratiques culturelles, laissant une place hégémonique à une série d'usages liés aux nouvelles technologies, et au premier chef aux pratiques centrées sur les nouvelles formes de consommation de musique et de films. Dans la liste fournie de ces écarts positifs à la moyenne des pratiques liées aux nouvelles technologies se distinguent aussi ceux ayant trait aux jeux vidéo (en ligne ou non), aux téléchargements non seulement de musique et de vidéos mais aussi de logiciels et d'images, aux formes les plus populaires du streaming (Youtube). Si cet ensemble évoque d'abord les usages populaires et «grand public» d'internet et des ordinateurs, il est intéressant de noter que des écarts positifs à la moyenne dans d'autres domaines complètent ce tableau d'une «culture jeune » (musiques rap et électroniques, accompagnées des radios Skyrock et Fun radio), et qu'en dehors d'un rejet accentué de l'opéra et du classique, peu d'écarts négatifs très significatifs ne marquent un comportement bien différent du comportement moyen s'agissant des formes plus classiques de pratiques culturelles.

Si la large place qu'y prennent les nouvelles technologies et la forte présence des moins de 40 ans (moins que précédemment, $83 \%$ contre $88 \%$ ) semble rapprocher la deuxième classe de la gauche du graphique $\left(\mathrm{C}_{2}\right)$ de celle qui précède, deux différences majeures marquent une distance significative à son modèle jeune. D’abord, aux côtés des mêmes usages massifs d'internet et de l'ordinateur (téléchargements divers, blogs...) apparaissent ici des écarts très significatifs à la moyenne d'autres usages plus culturels des nouvelles technologies : fréquentation d'internet liée aux préoccupations professionnelles, politiques, à la presse, au savoir et aux pratiques d'amateurs (documentation, dictionnaires, programmation, écriture, dessin, voyages, etc.). Quelques items évoquent, en complément de l'intégration aux nouveaux usages numériques des jeunes générations, des pratiques culturelles plus classiques (fréquentation des musées, du théâtre, des expositions d’art, pratique musicale, etc.) ou de générations un peu plus âgées (le rock prime ici sur le rap ou la musique électronique, la lecture de livres ne se limite pas aux mangas, la télévision signale plutôt l'émission sati- 
rique Les Guignols que l'émission de talk show La Méthode Cauet ou la téléréalité). Cette classe est plutôt celle de jeunes professionnels ou d'étudiants qui ne se distinguent pas seulement de la moyenne par la force de leurs pratiques numériques.

La troisième classe à gauche du dendrogramme $\left(\mathrm{C}_{3}\right)$ se caractérise par des pratiques numériques qui, sans être absentes de l'univers des pratiques distinguant cette classe de la moyenne, le sont à un rang inférieur de pouvoir explicatif pour la simple raison que toutes les pratiques culturelles qui viennent en tête de la liste relèvent des formes plus classiques de culture (expositions de peinture, musées, galeries, monuments, romans, essais, livres d'art, d'histoire, etc.). Et surtout, les pratiques numériques les plus significatives ici sont elles-mêmes en rapport avec ces pratiques de la culture : usage d'internet pour la presse, la documentation, l'accès aux musées, la politique. Les items socio-démographiques de la classe 3 les plus typiques évoquent plutôt la figure du cadre, d'âge moyen ou mûr, diplômé, ne regardant pas la télévision tous les jours, ou pour y voir Arrêt sur images, Striptease ou Les Mercredis de l'Histoire mais jamais La Méthode Cauet ou les jeux télévisés.

Dans la partie droite du graphique, la classe étiquetée $\mathrm{C}_{4}$ offre, à observer la liste des items qui y marquent les plus forts écarts à la moyenne, l'image d'un mélange entre de nombreuses «non-pratiques » (relevant tant des formes «numériques» que des formes traditionnelles) et une sur-fréquentation des formes populaires de la télévision (téléréalité, La Méthode Cauet, Super Nanny, etc.). Les modalités socio-démographiques qui sont nombreuses en tête de la liste permettent de préciser les caractéristiques de ceux qui partagent ce rapport aux pratiques culturelles. Le cœur de cette classe est en fait la mère de famille ayant encore de jeunes enfants, plutôt d'origine populaire, dont on peut faire l'hypothèse qu'elle n'a pas plus de temps ni de budget pour les pratiques culturelles «classiques » à l'extérieur (elle ne fréquente guère musées, théâtres et monuments, n'écoute pas de classique) que pour de longues connexions à internet. La télévision comme la radio (NRJ en tête) caractérisent cette manière-là de consacrer peu de temps à des pratiques culturelles explicites. 
Plus à droite sur le dendrogramme, la classe $\mathrm{C}_{5}$ est bien différente de la précédente car si ses membres ne s'y distinguent pas plus, et même encore moins, quant aux nouvelles pratiques numériques (les items «non» de ces domaines sont très nombreux avec une forte valeur test), ils privilégient en revanche les pratiques culturelles classiques, et notamment les formes les plus «didactiques» (musées, livres d'histoire, émissions culturelles à la télévision), et tout à l'inverse y rejettent le plus souvent la télévision de divertissement. Appartenant aux professions intermédiaires et à la catégories des cadres, encore actifs (tranche 40-59 ans), ceux qui constituent le cœur de cette classe ne sont pas forcément coupés du nouveau monde numérique (leurs foyers sont même un peu plus qu'en moyenne reliés à internet), mais celui-ci ne semble pas tenir une place importante dans leur vie (il est symptomatique que la seule occurrence d'un item positif en ce domaine dans la liste soit l'usage documentaire d'internet, comme s'il n'y avait là qu'un simple appoint aux pratiques plus traditionnelles).

Enfin, à la droite du dendrogramme, la classe C6, celle du tiers des plus éloignés des domaines culturels qui n’est définie que par des «non pratiques », et au premier rang par celles liées aux nouvelles technologies (plus de 90\% n'utilisent pas internet, cet item obtenant la plus forte valeur-test de l'analyse). Les modalités des non-pratiques apparaissent élevées pour tout ou presque: sorties culturelles ou de vie sociale, pratiques culturelles classiques - seule une émission de télévision de divertissement, Grand Cabaret, marque un écart significatif à la moyenne ( $57 \%$ la regardent ici contre $39 \%$ en moyenne). L'âge semble être un déterminant majeur de cette absence de pratiques $-47 \%$ ont plus de 65 ans - mais il cumule ses effets avec ceux de l'origine sociale et du faible bagage culturel - la plus forte valeur-test «socio-démographique » pour la classe 6 est celle de l'item croisé «[anciens] employés-ouvriers de niveau inférieur au baccalauréat et de plus de 60 ans ». 


\section{I. LES DOMINANTES CULTURELLES ET SOCIODÉMOGRAPHIQUES DES UNIVERS CULTURELS}

Le critère de sélection des modalités retenues dans le tableau 2 ci-après est, pour chaque domaine culturel et chaque variable socio-démographique, celui de la (ou des) modalité des variables socio-démographiques ou culturelles la plus élevée de la classe (pourcentages en colonne) quand celle-ci est elle-même significativement élevée par rapport aux valeurs de la modalité dans les autres classes (pourcentages en ligne).

De façon à ne pas induire un effet «profil» ou «portrait» tiré des classes et se prémunir d'une induction à généraliser les dominantes d'une classe à l'ensemble de ses membres, nous avons retenus les modalités de composition sociodémographique et de pratiques supérieures à $20 \%$ de la catégorie, seuil qui constitue en lui-même un critère sans doute très généreux pour qualifier une classe (qualifier une classe par une pratique qui concerne moins du quart de ses membres cède en effet à la logique généralisante, celle qu'on peut déceler, par exemple, dans le dispositionnalisme, celui de la théorie de l'habitus) ${ }^{18}$. C'est l'ensemble de ces traits qui caractérisent la classe dans une logique des proportions et non de portraits.

En relation avec l'analyse factorielle, la classification hiérarchique tend à se structurer, dans sa partie gauche (classe I à 3) puis droite (classe 4 à 6), selon une logique qui combine critère socioprofessionnel et critère de l'âge, faisant passer des plus jeunes aux plus âgés et des positions les plus basses aux plus élevées. On voit d'une part grandir, de la classe I à la classe 3, l'ensemble des

18 Où «la position sociale engendre des dispositions culturelles déterminées» (Coulangeon, 2004, p. 65), à savoir la transposabilité d'une appétence pour un objet $x$ à l'ensemble des objets «homologues». La logique dispositionnaliste structure très nettement - en s'autorisant des «écarts à l'indépendance» des pratiques (Cogneau, 1990) - l'interprétation de l'enquête de 1988-1989 du ministère de la Culture. Denis Cogneau y lit les loisirs culturels en termes «d'univers de loisir possibles»: «si vous allez souvent au musée vous allez sans doute aussi au théâtre et au restaurant» (ibid., p. 179), puisque «les cartes culturelles ne délimitent pas étroitement des noyaux de pratiquants réguliers. Elles indiquent des associations de cumuls de pratiques possibles, qu'ils soient réels [...] ou potentiels (ibid, p. |8I). La description construit une figure du «cumulard potentiel» (ibid.). C'est nous qui soulignons. Voir Bourdieu, 1984, p. 134. 
pratiques culturelles concernant une part majoritaire des membres de la classe, d'autre part une distinction apparaître entre des «goûts » générationnels (par exemple, musiques électroniques et rap pour la classe I, puis rock et électronique pour la classe 2, enfin classique-jazz-opéra pour la classe 3) selon une logique générationnelle qui valorise plus ou moins les différents genres culturels sans qu'aucune d'entre les classes ne les pratique tous, y compris la classe 3 des forts pratiquants. Une logique d'âge (et sans doute générationnelle) distingue les vingtenaires et les plus jeunes, les trentenaires et les quadragénaires au sein même d'une population diplômée ou étudiante.

De la classe 4 à la classe 6, les dominantes culturelles se font rares et les inappétences plus nettes (notamment celle de la classe 4 pour l'opéra et celle des classes 5 et 6 pour la musique rap). L'univers sociologique est celui des catégories populaires ou des professions intermédiaires et il voit se distinguer aussi les plus jeunes (classe 4) et les plus âgés (classe 6). Comme nous l'avons vu plus haut dans la description des classes, la télévision et une pratique des musées caractérisent respectivement les classes 4 et 5 les plus actives en matière culturelle de cet ensemble des Français.

Comme on le voit, nous voudrions y insister, il n'y a à chaque fois que quelques modalités de variables culturelles (de pratiques) ou de variables socio-démographiques qui caractérisent nettement - par exemple au-delà de $50 \%$-, telle ou telle classe d'ensemble de pratiques, même parmi celles qui typent la classe par leur écart à la moyenne (Glevarec et Pinet, 20I3). Rien n'autorise à extrapoler, à partir de taux souvent minoritaires. À la façon dont B. Lahire (2004, p. 137) a pu le souligner en termes de surestimation des pratiques «légitimes » des catégories supérieures, notre perspective consiste davantage à s'en tenir au réel statistique, modeste, des proportions de pratiquants et à soutenir une hypothèse d'éventails circonscrits de genres pratiqués, dans un environnement culturel dense, marqués, on l'a vu, par un effet décisif du facteur générationnel. 
Tableau 2. Les dominantes culturelles et sociodémographiques des univers culturels (i.e. classes)

\begin{tabular}{|c|c|c|c|c|c|c|c|c|c|c|c|c|c|c|c|}
\hline $\begin{array}{l}0 \\
\text { 岁 } \\
\underline{y}\end{array}$ & 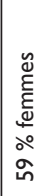 & 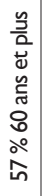 & & $\begin{array}{l}\text { 岀 } \\
\stackrel{0}{\circ} \\
\stackrel{n}{n}\end{array}$ & & $\begin{array}{l}\frac{\tilde{\omega}}{\hat{o}} \\
\frac{0}{0} \\
\frac{\hat{\varepsilon}}{0} \\
\frac{0}{0} \\
\frac{m}{m}\end{array}$ & 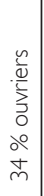 & 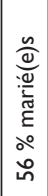 & 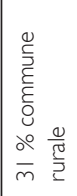 & 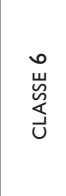 & 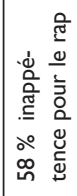 & 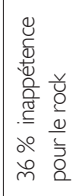 & & & \\
\hline 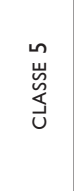 & & 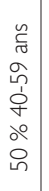 & & 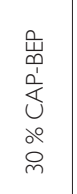 & & 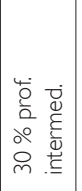 & & 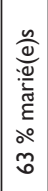 & & 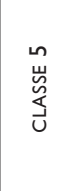 & 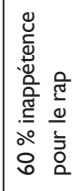 & & & & \\
\hline 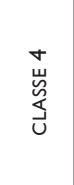 & & 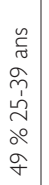 & & 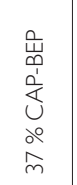 & & 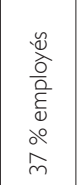 & & & & 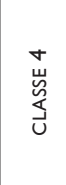 & 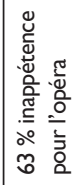 & & & & \\
\hline 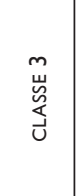 & & 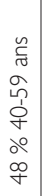 & & 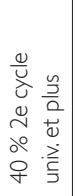 & & 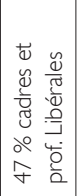 & & 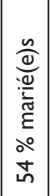 & 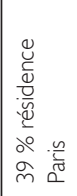 & 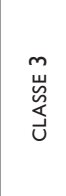 & 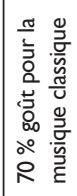 & 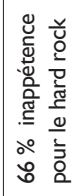 & 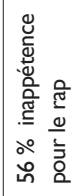 & 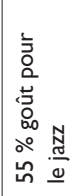 & 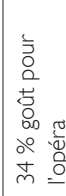 \\
\hline 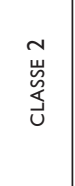 & 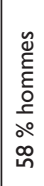 & 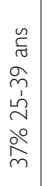 & & 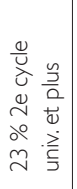 & & 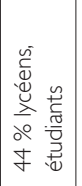 & & 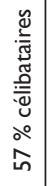 & & 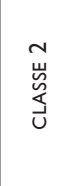 & 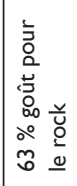 & 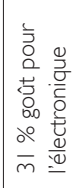 & & & \\
\hline 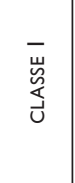 & 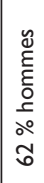 & 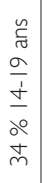 & 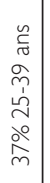 & 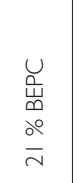 & 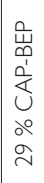 & 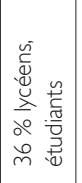 & 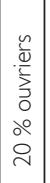 & 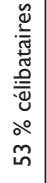 & & 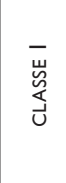 & 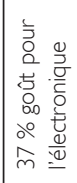 & 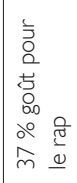 & & & \\
\hline 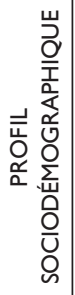 & 崫 & $\underline{\widetilde{U}}$ & & 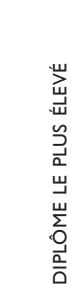 & & 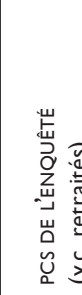 & & 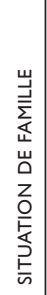 & 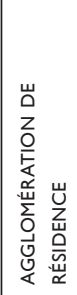 & 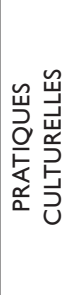 & & & $\begin{array}{l}\frac{\mathrm{U}}{\mathrm{O}} \\
\frac{\mathrm{v}}{\mathrm{N}} \\
\frac{\mathrm{s}}{\mathrm{T}}\end{array}$ & & \\
\hline
\end{tabular}




\begin{tabular}{|c|c|c|c|c|c|c|c|c|c|c|c|c|}
\hline 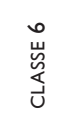 & & & & & & & & & & & & \\
\hline 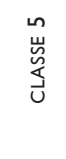 & & & & & 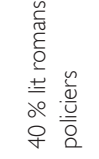 & & & & 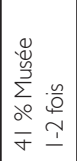 & & & \\
\hline 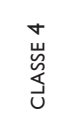 & & & & & & & & & & & & \\
\hline 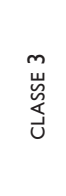 & 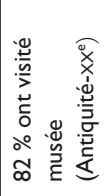 & 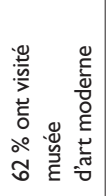 & 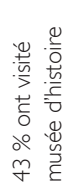 & 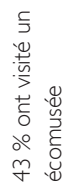 & 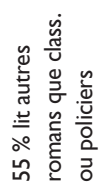 & 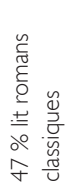 & 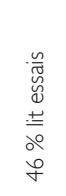 & 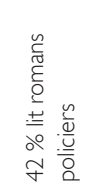 & 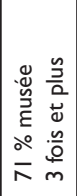 & 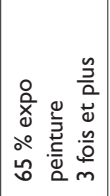 & 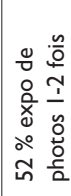 & 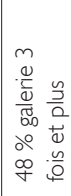 \\
\hline 岀 & 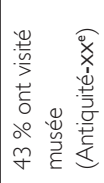 & & & & 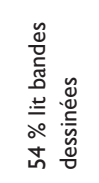 & 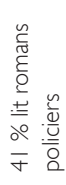 & 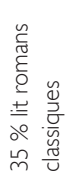 & 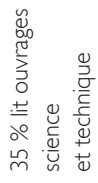 & 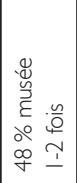 & 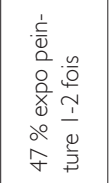 & & \\
\hline $\begin{array}{l}\bar{w} \\
\text { Ș } \\
\text { U. }\end{array}$ & & & & & & & & & & & & \\
\hline 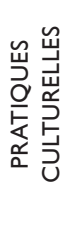 & & 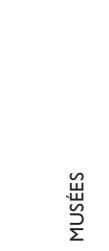 & & & \multicolumn{4}{|c|}{ 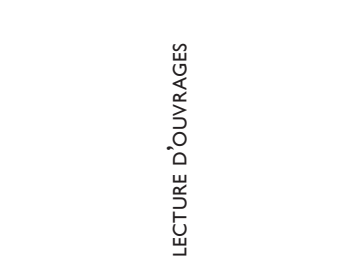 } & \multicolumn{4}{|c|}{ 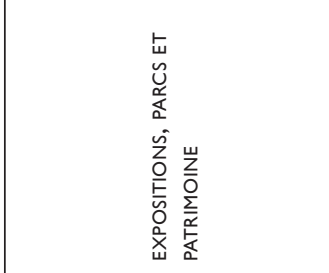 } \\
\hline
\end{tabular}




\begin{tabular}{|c|c|c|c|c|c|c|c|c|}
\hline 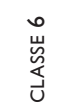 & & & & & & & & \\
\hline $\begin{array}{l}\text { n } \\
\text { 岀 } \\
\text { Ș }\end{array}$ & & & & & & & & \\
\hline 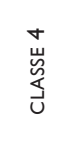 & 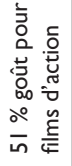 & 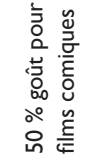 & & & & & & \\
\hline 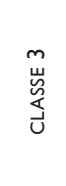 & 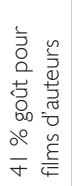 & 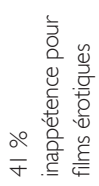 & 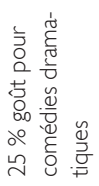 & 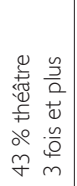 & 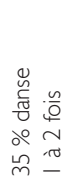 & $\begin{array}{ccc} & \\
& \frac{n}{0} \\
\frac{0}{0} & \frac{3}{0} \\
0 & 0 \\
0 & 0 \\
00 & \frac{n}{0} \\
0 & \frac{0}{0} \\
m & -\end{array}$ & 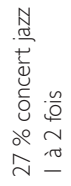 & 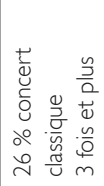 \\
\hline $\begin{array}{l}\text { U } \\
\text { 岁 } \\
\text { U }\end{array}$ & & & & 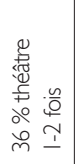 & 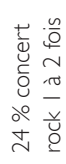 & & & \\
\hline $\begin{array}{l}\bar{u} \\
\text { 岁 } \\
\text { U }\end{array}$ & 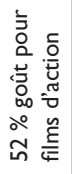 & 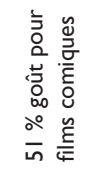 & & & & & & \\
\hline 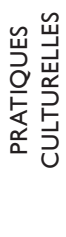 & & $\sum_{\frac{\mathbb{U}}{Z}}^{\frac{\pi}{U}}$ & & & & 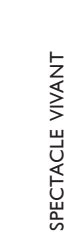 & & \\
\hline
\end{tabular}

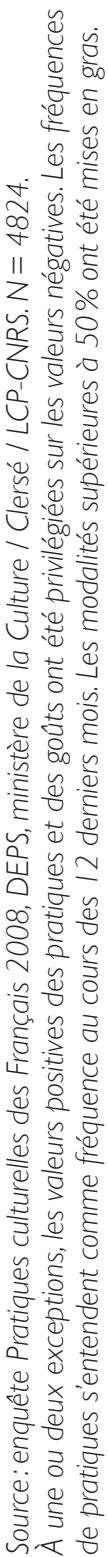


L'usage fait ici de la méthode d'analyse des correspondances restreint ses conclusions à l'identification d'archipels de goûts. En appui d'un modèle de la diversification culturelle qui apparaît plus réaliste parce que plus nettement descriptif (Glevarec et Pinet, 20I3), il ne vise pas à interpréter des classes de pratiques comme des profils culturels actualisés ou potentiels. Les conclusions ne versent pas dans le dispositionnalisme culturel parce qu'il s'agit de rendre compte du fait que les pratiques sont devenues, pour une part de leur explication, générationnelles, c'est-à-dire culturelles. C'est le fait d’appartenir à telle génération qui explique pour partie les pratiques actuelles des individus et non (plus) exclusivement le capital culturel possédé ou hérité. Si une telle méthodologie quantitative ne permet pas de saisir le sens vécu des pratiques culturelles, elle autorise à soutenir une autre structuration des pratiques que l'argument premier de La Distinction au profit d'un axe majeur de l'âge / effet de l'appartenance historique à une génération.

\section{CONCLUSION \\ Le capital culturel face à la variable générationnelle}

L’analyse des correspondances multiples menée sur les données 2008 de l'enquête sur les pratiques culturelles des Français confirme que si l'axe de la stratification sociale des pratiques culturelles demeure premier dans la structuration des pratiques culturelles, le second, celui de la différenciation selon les possessions culturelles ou économiques mise en évidence par P. Bourdieu, est dorénavant un axe de l'âge. La place prise par cette dimension constitue en soi un tournant culturel s'il s'agit de constater qu'elle recouvre la génération; une variable de nature culturelle - l'ensemble des biens fréquentés et des goûts formés par chaque génération historique - explique une part des différents univers de pratiques.

Parler de tournant culturel est la façon de rendre compte de ce fait sociologique et historique que les biens culturels eux-mêmes seraient devenus des variables déterminantes et explicatives - sous la forme de la génération culturelle - de la distribution des pratiques en France en tant que second facteur après la stratification sociale. Sur le second axe, la notion de «capital culturel» n’a plus le même sens. Dans l'acception de P. Bourdieu, elle désigne un capital, comptable 
(possédé plus ou moins), universel (valant sur un marché unifié), transformable (en d'autres capitaux), hérité (en famille) ou acquis (à l'école); dans son acception générationnelle, capital culturel perd ce sens premier parce que la différence observée n'est plus quantitative (avoir ou pas telle pratique) mais qualitative (appartenir à la «culture d'écran» ou de «l'imprimé» (Donnat, 2009, p. 223) ou aimer la musique classique ou la musique rock, etc.). Les «cultures générationnelles » issues de l'analyse sur les effets d'âge et de génération sur les pratiques culturelles et médiatiques depuis trente ans en France (BIPE/DEPS, 2007), non seulement remplacent le couple divorcée entre une «culture classique» et une «culture médiatique » au profit d'une pluralisation des «cultures», mais oblige à redéfinir la place de la culture et du «capital culturel» comme variable expliquée ou expliquant. Le facteur que dénote les variations générationnelles de pratiques culturelles est davantage lié à un capital d'expériences culturelles passées qu’à un capital culturel comme compétences qu'elles soient conçues comme compétences en culture classique ou scolaire ou comme compétences génériques applicables à des domaines culturels nouveaux.

\section{BIBLIOGRAPHIE}

BENNETT T., SAVAGE M., SILVA E. B., WARDE A., GAYO-CAL M., WRIGHT D. (eds.), 2009, Culture, Class, Distinction, Oxon / New York, Routledge.

BIPE/DEPS, 2007, Approche générationnelle des pratiques culturelles et médiatiques,

Paris, DEPS, ministère de la Culture et de la Communication:

http://www.culture.gouv.fr/deps.

BOURDIEU P., 1979, La Distinction. Critique sociale du jugement, Paris, Minuit.

-, 1984, Questions de sociologie, Paris, Minuit.

-, SAINT-MARTIN M., 1976, «Anatomie du goût», Actes de la recherche en sciences sociales, 2-5, p. 2-8I.

COGNEAU D., 1990, «Les pratiques culturelles dans l'espace des loisirs», in Les Pratiques culturelles des Français, O. Donnat et D. Cogneau (éds), 1973-1989, Paris, La Découverte/La Documentation française, p. 143-286. 
COULANGEON P., 2004, «Classes sociales, pratiques culturelles et styles de vie. Le modèle de la distinction est-il (vraiment) obsolète?», Sociologie et sociétés, 36-I, p. 59-85.

COULANGEON P., LEMEL Y., 2007, «Is "distinction” really outdated? Questioning the meaning of the omnivorization of musical taste in contemporary France», Poetics, 35-2/3, p.93-111.

-, 2010, «Bourdieu's legacy and the class-status debate on cultural consumption: musical consumption in contemporary France», in Social Status and Cultural Consumption, T.W. Chan (ed.), Cambridge, Cambridge University Press, p. 84-108.

DONNAT O., 1994, Les Français face à la culture: de l'exclusion à l'éclectisme, Paris, La Découverte.

-, 1998, Les Pratiques culturelles des Français. Enquête 1997, Paris, La Découverte/La Documentation française.

-, 2004, «Les univers culturels des Français», Sociologie et sociétés, 36-I, p. 88-103.

-, 2005, «La connaissance des publics et la question de la démocratisation», Culture et Recherche, 106-107, p. 16-17.

-, 2009, Les Pratiques culturelles des Français à l'ère numérique. Enquête 2008, Paris, La Découverte/Ministère de la Culture et de la Communication.

-, 2011, «Pratiques culturelles, 1973-2008. Dynamiques générationnelles et pesanteurs sociales», Culture Études, Département des études, de la prospective et des statistiques, 7.

-, COGNEAU D., 1990, Les Pratiques culturelles des Français, 1973-1989, Paris, La Découverte/La Documentation française.

-, TOLILA P., 2003, Le(s) Public(s) de la culture, Paris, Presses de Sciences Po.

GLEVAREC H., 2013, La Culture à l'ère de la diversité. Essai critique trente ans après La Distinction, Paris, Éditions de l'Aube.

GLEVAREC H., PINET M., 2009, «La "tablature" des goûts musicaux: un modèle de structuration des préférences et des jugements», Revue française de sociologie, 50-3, p. 599-640.

-, 2012, «Tablatures of Musical Tastes in contemporary France: Distinction without intolerance», Cultural Trends, 21-1, p.67-88. 
-, 2013, «De la distinction à la diversité culturelle, Eclectismes qualitatifs, reconnaissance culturelle et jugement d'amateur», L'Année sociologique, 2.

GRIGNON C., PASSERON J.-C., 1989, Le Savant et le Populaire, Paris, Gallimard-Seuil.

LAHIRE B., 1996, «Risquer l'interprétation. Pertinences interprétatives et surinterprétations en sciences sociales», Enquête, p. 61-87.

-, 2004, La Culture des individus. Dissonances culturelles et distinction de soi, Paris, La Découverte.

LE ROUX B., ROUANET H., 20I0, Multiple Correspondence Analysis. Series: Quantitative Applications in the Social Sciences, London, Sage.

MERCKLÉ P., 2010, «Le modèle de la distinction est-il (déjà) pertinent? Premiers résultats de l'enquête longitudinale sur les pratiques culturelles des enfants et des adolescents», Colloque international «30 ans après La Distinction », Paris, 4 novembre 20I0: http://socio.ens-lyon.fr/merckle/merckle_communications_2010_distinction.pdf.

OCTOBRE S., 2004, Les Loisirs des 6-14 ans, Paris, La Documentation française.

-, DETREZ C., MERCKLÉ P., BERTHOMIER N. (éds.), 20II, L'Enfance des loisirs. Trajectoires communes et parcours individuels de la fin de l'enfance à la grande adolescence, Paris, La Documentation française.

ROOSE H., VAN EIJCK K., LIEVENS J., 2012, «Culture of distinction or culture of openness? Using a social space approach to analyze the social structuring of lifestyles», Poetics, 40-6, p. 49|-513.

ROUANET H., ACKERMANN W., LE ROUX B., 2000, «The Geometric Analysis of Questionnaires. The Lesson of Bourdieu's La Distinction», Bulletin de méthodologie sociologique, 65, p.5-15. 